1 Title:

2 Clinical characteristics and high resolution retinal imaging of retinitis pigmentosa caused by $R P 1$ gene variants

4 Running title: Clinical and AO findings in RP1-ARRP

5 Authors: Shinji Ueno ${ }^{1}$, Yoshito Koyanagi ${ }^{1,2}$, Taro Kominami ${ }^{1}$, Yasuki Ito ${ }^{1}$, Kawano Kenichi ${ }^{1}$, 6 Koji M Nishiguchi $^{3}$, Carlo Rivolta ${ }^{4,5,6}$, Toru Nakazawa ${ }^{3}$, Koh-Hei Sonoda ${ }^{2}$ and Hiroko Terasaki ${ }^{1}$ 7

\title{
8 Institutions:
}

$9 \quad{ }^{1}$ Department of Ophthalmology, Nagoya University Graduate School of Medicine, Nagoya, Japan

102 Department of Ophthalmology, Graduate School of Medical Sciences, Kyushu University, Fukuoka, 11 Japan

$12{ }^{3}$ Department of Ophthalmology, Tohoku University Graduate School of Medicine, Sendai, Japan

$13{ }^{4}$ Institute of Molecular and Clinical Ophthalmology Basel (IOB), Basel, Switzerland

$14 \quad{ }^{5}$ University of Basel, Basel, Switzerland

$15{ }^{6}$ Department of Genetics and Genome Biology, University of Leicester, Leicester, UK

*Correspondence: Shinji Ueno, M.D., Ph.D., Department of Ophthalmology, Nagoya University

19 Graduate School of Medicine, 65 Tsuruma-cho, Showa-ku, Nagoya 466-8550, Japan

20 Tel: +81-52-744-2277, Fax: +81-52-744-2278

21 E-mail: ueno@med.nagoya-u.ac.jp

22 Word count; (abstract) $241 \quad$ (main text) 3316

23 Total number of references; 26

24 Total number of figures; 8 figures

25 Total number of table; 1 
28 Purpose: To report the clinical course and high resolution images with autosomal recessive retinitis

29 pigmentosa (RP) associated with a variant of the $\underline{R P 1}$ gene (c.4052_4053ins328/p.Tyr1352Alafs*9;

$30 \mathrm{~m} 1$ ). This variant has been found to be a high frequency founder variant in Japanese RP patients.

31 Study Design: Retrospective case series

32 Methods: Nine patients from 5 unrelated Japanese families were studied. Five patients had the $\mathrm{m} 1$

33 variant homozygously, and 4 patients had the $\mathrm{m} 1$ variant compound heterozygously with another

34 frameshift variant, (c.4196delG/p.Cys1399Leufs*5). Ophthalmic examinations including adaptive

35 optics (AO) fundus imaging were performed periodically.

36 Results: The fundus photographs, fundus autofluorescence (FAF) images, and optical coherence

37 tomographic (OCT) images indicated severe retinal degeneration involving the macula even at a

38 young age (20s) in all the patients. The areas of surviving photoreceptor in the central macula were

39 seen as hyper-autofluorescent regions in the FAF images and preserved outer retinal structure in the

40 OCT images, and they were identifiable in the AO fundus images in 8 eyes. The borders of the

41 surviving photoreceptor areas were surrounded by hyporeflective clumps, presumably containing melanin, and the size of these areas decreased progressively during the 4-year follow-up period. The

43 disappearance of the surviving photoreceptor areas was associated with complete blindness.

44 Conclusion: Patients with RP associated with the $\mathrm{m} 1$ variant have a progressive and severe retinal 
46 imaging can provide a more precise pathological progress of the retinal degeneration.

47 
48 Keywords: Autosomal recessive retinitis pigmentosa; RP1; Adaptive optics images; Fundus

49 autofluorescence

50

51

52 
54 Retinitis pigmentosa (RP) represents a heterogeneous group of retinal disorders that is characterized

55 by a progressive degeneration of both the rod and cone photoreceptors bilaterally. The $R P 1$ gene was

56 the first gene identified to cause autosomal dominant $\operatorname{RP}(\operatorname{adRP})[1,2]$, and it was later found to also

57 cause autosomal recessive RP (arRP) [3-6]. Recently, $R P 1$ was reported to be associated with

58 autosomal recessive macular dystrophy and autosomal recessive cone-rod dystrophy $[7,8] . R P 1$

59 encodes a multimodular protein of 2156 amino acids, and it is a member of the doublecortin family.

$60 R P 1$ is present in the ciliary axoneme of both rods and cones [9]. Mutations in $R P 1$ is associated

61 with a decrease in the best-corrected visual acuity (BCVA) which is due to a ciliopathic phenotype

62 caused by an abnormal stacking of the outer segment discs of rods and cones [10].

63 To date, at least 170 mutations have been reported in $R P 1$, and most of these mutations cluster

64 within its last exon, exon 4, cumulatively accounting for approximately for $5.5 \%$ of all adRP and

65 up to $4.5 \%$ of all autosomal arRP cases $[5,11]$.

66 Our earlier study by whole-genome sequence screening of Japanese patients identified a new,

67 unusual mutation consisting of an insertion of a mobile Alu element in exon 4 of the $R P 1$ gene

68 (c.4052_4053ins328/p.Tyr1352Alafs*9; m1) [12]. This $\mathrm{m} 1$ insertion variant causes a disruption of

69 the reading frame by introducing 328 additional nucleotides and a premature termination codon in

70 the canonical $R P 1$ coding sequence. Additional targeted screening for the $\mathrm{m} 1$ variant in 330 

patients. However, precise phenotypic analyses were not performed in these earlier studies. Thus, the purpose of this study was to determine the pattern of progression of the retinal degeneration in arRP patients with the $\mathrm{m} 1$ variant of the $R P 1$ gene using adaptive optics (AO) fundus imaging.

This was an observational case series conducted at Nagoya University Hospital. The study protocol adhered to the tenets of the Declaration of Helsinki and was approved by the Institutional Review obtained from all participants. We studied 9 patients from 5 unrelated families in whom the $\mathrm{m} 1$ mutation had been identified.

84 Genetic analyses. In all families, genomic DNA was extracted from peripheral lymphocytes according to standard procedures. The genetic data of patients NA0039, NA1039, NA0070, NA0201,

86 NA1201, and NA0209 were obtained earlier by whole genome sequencing and direct sequencing

87 [12].The $\mathrm{m} 1$ and another frameshift variant (c.4196delG/p.Cys1399Leufs*5, m2) in patients

88 NA1048 and NA1201 and some of the unaffected family members were analyzed in this study. A 
pair of primers was designed to screen for the presence of the $\mathrm{m} 1$ and $\mathrm{m} 2$ variants in exon 4 of the

91 TTCTGCTTCTTTTTCACTTAGGC- ${ }^{\prime}$. The presence of $\mathrm{m} 1$ variant was confirmed from the products were purified and both strands of the gene were sequenced with an automated sequencer

\section{4 (Fasmaq, Atsugi, Kanagawa, Japan).}

96 Clinical Evaluations. Clinical data were obtained from the medical records. All patients had visual acuity measurements, Goldmann perimetry, conventional fundus photographs, and spectral-domain optical coherence tomography (SD-OCT, Spectralis; Heidelberg Engineering, Heidelberg, Germany). Fundus autofluorescence (FAF) imaging was performed with an ultra-widefield imaging device (Optos P200Tx; Optos, Dunfermline, UK) in 8 patients. camera (rtx1, Imagine Eyes, Orsay, France). This system was used in an earlier investigations to obtain images of individual cone photoreceptors [13-16]. Montage images were constructed within $6^{\circ}$ of the fovea using the i2k Retina software (DualAlign LLC, Clifton Park, NY, USA). The 
107 manually as described[13,15,16]. The area of analysis was $80 \times 80$ pixels or approximately 65

$108 \mu \mathrm{m} \times 65 \mu \mathrm{m}$ at $1^{\circ}$ and $3^{\circ}$ from the fovea on the nasal side and $3^{\circ}$ from the fovea on the temporal

109 side. To measure the size of surviving photoreceptor area, the processed images were analyzed with

110 the ImageJ software (version 1.48; the National Institutes of Health, Bethesda, MD, USA). The

111 axial length (AL) was obtained by the IOL Master (Carl Zeiss Meditec, Dublin, CA), and the AL

112 was used to adjust for the different degrees of magnification caused by differences in the AL.

113 The decimal BCVA was converted to the logarithm of the minimum angle of resolution (logMAR)

114 units for the statistical analyses. Counting fingers (CF), hand motion (HM), light perception (LP),

115 and no light perception, were designated as $1.85,2.30,2.80$, and $2.90 \operatorname{logMAR}$ units, respectively.

117 Results

118 Nine affected patients from 5 families with a clinical diagnosis of RP and harboring $\mathrm{m} 1$ variants

119 were studied. Five patients from 3 families had the $\mathrm{m} 1$ variant homozygously and 4 patients from 2

120 families had the $\mathrm{m} 1$ variant compound heterozygously with another frameshift variant

121 (c.4196delG/p.Cys1399Leufs*5, m2). The pedigrees of the 5 families are presented in Figure 1. All

122 five families were originally from Japan, and any mixture with other ethnicities was not reported.

123 Autosomal recessive family history was reported in 4 families and sporadic case was reported in one 
124 family (Family \#3). The parents of the patients were not affected even though they have the $\mathrm{m} 1$ or

$125 \underline{m 2}$ variant heterozygously.

126 The demographics of the clinical characteristics are presented in Table. The mean age was $17.9 \pm$

1276.2 years with a range of 8 to 27 years at the initial examination and that was $30.4 \pm 6.5$ years with

128 a range of 19 to 39 years at the last examination. The mean and median observation period was

12912.2 years and 13 years respectively. The initial symptom of all patients was night blindness and 8

130 patients experienced it from their childhood, while one patient (NA0209) first noted the night

131 blindness at age 15 years.

132 Visual acuity.

133 Even at the initial visit, the BCVA was worse than 1.0 (decimal visual acuity) in 8 of the patients.

134 BCVA was worse than 0.3 in the better eye in 5 patients at the last examination. The visual fields

135 were severely constricted even at a young age, and most of the patients had paracentral scotomata

136 with peripheral constriction or a central island. Electroretinograms (ERGs) were barely

137 recordable in 2 patients and non-recordable in the remaining 6 patients (ERG data of NA1039 was

138 unavailable.). These findings indicated panretinal degeneration in all of the patients.

139 The course of the decrease in the BCVA of each patient is shown in Figure 2. Due to the early

140 involvement of the macula, the BCVA had decreased to worse than 0.1 (decimal), which is 1.0

$141 \log$ MAR units, in at least one eye by the thirties. The BCVA tended to decrease gradually after 
142 initial visit. Then the BCVA deteriorated to worse than 0.1 (decimal) within a relatively short time

143 in patients NA0039, NA0201, NA1209, and NA1201 (Fig. 2).

\section{Fundus images.}

146 Fundus photographs were obtained from all 9 patients (Fig. 3 ), and ultra-widefield FAF images

147 were obtained from 8 patients (Fig. 4). All patients except NA0209 had severe degeneration

148 accompanied by bone-spicule pigmentation around the arcade vessels and macular degeneration.

149 FAF showed patchy and coalescent hypo-autofluorescent areas at the posterior pole which

150 indicated atrophy of the retinal pigment epithelium (RPE). Fine hypo-autofluorescent patchy areas

151 were present in the peripheral retina in all cases except NA0209. The fundus and FAF of case

152 NA0209 appeared to be a much milder phenotype compared to those of the other patients; fundus

153 showed minimum macular abnormalities, FAF showed hyperreflectivity in macular area, and small

154 patchy hypo-autofluorescense at the fovea(Figure 5).

155 OCT images of all the cases are shown in Figure 6. The OCT images showed abnormalities in the

156 outer retina, e.g., disrupted ellipsoid zone (EZ) and thinning of the outer nuclear layer. The EZ was

157 totally absent in all cases, except case NA0209, and several fine hyperreflective structures, like

158 tubulations, were found on the RPE in most of the eyes as shown by yellow arrows in Figure 6. 
161 The clinical manifestations of case NA0209 were much milder than those of the other patients; the

162 patient had no bone-spicule pigmentation, fewer hypo-autofluorescent patchy areas, and relatively

163 preserved BCVA. The changes of the FAF and OCT images of case NA0209 during a 5-year

164 follow-up period are shown in Figure 5. FAF showed an increase in the number of fine

165 hypo-autofluorescent patchy spots around the arcade vessels and the fovea. Similar features were

166 found in the eyes of his sister (case NA1209 in Fig. 4). In addition, OCT showed a severe reduction

167 of retinal thickness with the central retinal thickness reduced from $163 \mu \mathrm{m}$ to $131 \mu \mathrm{m}$. These results

168 indicated a progressive retinal degeneration both in the macular and peripheral retina. The AO

169 fundus images at 21-years-of-age showed cone mosaics although they were sparsely distributed

170 compared to those of normal control eyes (Fig. 5B). The cone densities at the $1^{\circ}$ and $3^{\circ}$ from the

171 fovea on the nasal side were $11231 / \mathrm{mm}^{2}$ and $7718 / \mathrm{mm}^{2}$ respectively, and that at $3^{\circ}$ on the temporal

172 side was $8984 / \mathrm{mm}^{2}$. As reference, the of cone densities in a normal control eye at $1^{\circ}$ and $3^{\circ}$ from the

173 fovea on the nasal side and $3^{\circ}$ on the temporal side were $31042 / \mathrm{mm}^{2}, 23283 / \mathrm{mm}^{2}$, and $24333 / \mathrm{mm}^{2}$,

174 respectively.

175

176 Analyses of $\mathrm{AO}$ fundus images of surviving photoreceptor areas. 
177 We obtained the AO fundus images of 8 patients except case NA1039. The images of one case

178 (NA0048) were difficult to analyze because the quality was poor due to unsteady fixation. In general,

179 AO fundus imaging has been used to detect and count the number of photoreceptors in eyes with

180 hereditary retinal disorders. However, cone mosaics were detected in the AO images only in the eyes

181 of case NA0209 (Fig. 5). Therefore, we focused on other retinal structures in the AO images.

182 The changes of AO images of the right eye of case NA0201 during a 4-year follow-up period are

183 shown in Figure 7. Isolated, small, and deeply-colored areas were detected in the fundus

184 photographs corresponding to the areas of the hyper-autofluorescence in the FAF images of the

185 central macula. The remaining hyperreflective structures on the RPE in OCT images corresponded

186 to the hyper-autofluorescent areas in the FAF images. The AO fundus images precisely identified

187 the areas of hyper-autofluorescence in the FAF images, which presumably represented island-like

188 residual photoreceptor areas and named "photoreceptor islands". In contrast to their surrounding

189 surfaces, the areas were homogenous even though the cone mosaics were invisible. The edges of

190 the islands were partially covered by hyporeflective clumps (HRCs). HRCs were observed not only

191 at the border of the islands but also around the photoreceptor islands. Higher magnified images

192 showed that the small HRCs participated in the formation of the larger HRCs (Fig. 7 top right). The

193 size of photoreceptor island appeared smaller and less homogenous after 4 years which made their

194 borders unclear. The mean area of the residual photoreceptor island measured in AO fundus images 
was reduced from $6.0 \times 10^{5} \mu \mathrm{m}^{2}$ in 2013 to $4.3 \times 10^{5} \mu \mathrm{m}^{2}$ in 2015 , and to $2.9 \times 10^{5} \mu \mathrm{m}^{2}$ in 2017 .

196 The BCVA decreased from 0.13 (decimal) in 2013 to 0.06 in 2015, and 0.02 in 2017. The

197 corresponding areas of the OCT images showed a decrease in retinal thickness due to a reduction of

198 the thickness of the outer nuclear layer (ONL, Fig. 7).

199 Similar AO findings were found in other 7 eyes; the right eye (Rt) and left eye (Lt) of NA1048,

200 NA0201Lt, NA1201Lt, NA0039Lt, NA0070Rt, and NA1209Lt. However, the quality of the AO

201 images of NA201Lt (BCVA; 0.1) and 1209Lt (BCVA; 0.02) were poor, and the borders of the

202 photoreceptor islands were too indistinct to analyze. In 5 eyes (NA0039Rt, NA1201Rt, NA0070Lt,

203 NA1209Rt, and NA1209Rt eyes) the photoreceptor islands could not be detected in the FAF or in

204 the AO images presumably due to a complete loss of the residual photoreceptor areas. The BCVA in

205 these eyes was close to total blindness and ranged from $\underline{\mathrm{LP}}$ to 0.05 (decimal). $\underline{\text { In addition to the AO }}$

206 image of NA0201Rt, those of NA1201Lt, NA0039Lt, and NA0070Rt eyes were followed for about

207 four years, and the baseline images are shown in Figure 8. The OCT images showed that the fine

208 structures appeared like outer retinal tubulations on the RPE in the corresponding areas. The course

209 of the reduction in the size of the photoreceptor islands measured in AO fundus images and BCVA

210 in NA0039Lt, NA0201Rt, NA1201Lt, and NA0070Rt are shown in Figure $\underline{8 B}$. During the 4 year of

211 follow-up period, the size of the photoreceptor island in case NA1201Lt was reduced from $8.2 \mathrm{x}$

$21210^{5} \mu \mathrm{m}^{2}$ to $4.8 \times 10^{5} \mu \mathrm{m}^{2}$, however the BCVA was maintained at 0.5 (decimal). On the other hand, 
213 the size of photoreceptor islands in the other 3 eyes (NA0039Lt, NA0201Rt, and NA0070Rt)

214 decreased to less than $3.0 \times 10^{5} \mu \mathrm{m}^{2}$ with a reduction of the BCVA to $<0.1$ (decimal). The AO

215 images of NA1048Rt and Lt were obtained only once and the sizes were $12.1 \times 10^{5} \mu \mathrm{m}^{2}$ and $25.1 \mathrm{x}$

$21610^{5} \mu \mathrm{m}^{2}$ respectively (images not shown) with relatively preserved BCVA ( 0.5 in both eyes).

\section{$218 \quad$ Discussions}

219 Several $R P$ lvariants have been reported to cause adRP or arRP in Japanese patients [6, 7, 17-19].

220 The $\mathrm{m} 1$ variant had not been identified as a causative variant of arRP in a large Japanese RP cohort

221 study using targeted resequencing [18,19], although the $\mathrm{m} 1$ variant was present in $4.5 \%$ of RP

222 patients in our recent study [12]. The reason for this discrepancy might be that the $\mathrm{m} 1$ variant was

223 missed by targeted resequencing because the insertion of 328 nucleotides was too large to be

224 detected. Indeed, the $\mathrm{m} 1$ variant was first detected by Whole-Genome Sequence screening [12], and

225 our earlier targeted resequencing could not detect the $\mathrm{m} 1$ variant in case NA0201 although the m2

226 variant was detected [19]. This indicated that the $\mathrm{m} 1$ variant might be the cause of patients whose

227 causative genes had not been identified by targeted resequencing, especially in early onset and

228 severe RP cases with macular involvement.

229 Patients with the $\mathrm{m} 1 / \mathrm{m} 1$ and $\mathrm{m} 1 / \mathrm{m} 2$ variants appeared to have no significant differences in the

230 course of retinal degeneration; both genotypes shared phenotypic characteristics including severe 
231 retinal degeneration with early macular involvement. In addition, clinical courses of our patients

232 resemble that of ARRP caused by other frameshift variants in RP1.Thus we speculate the disease

233 mechanism of $\mathrm{m} 1$ and $\mathrm{m} 2$ is the complete loss of function due to nonsense-mediated mRNA decay.

234 The early BCVA loss was caused by macular degeneration at a younger age which can be explained

235 by the expression of the protein encoded by $R P 1$ in the ciliary axoneme of both rods and cones [9,

236 10].

237 Our results indicated that all of our patients, except case NA0209, had an early onset of impaired

238 vision that occurred during the first decade of life. This was followed by a severe decrease of the

239 BCVA in the second and third decade which has been reported as a phenotype of arRP caused by

$240 R P 1$ variants $[4,6,7,11,20]$. Only case NA0209 had a relatively late onset retinal degeneration

241 compared to the other patients including his sibling. However, even in this patient, OCT showed a

242 progressive reduction of the retinal thickness and FAF showed a similar retinal degeneration pattern

243 as his sister. In addition, fundus AO images indicated that the cone density was reduced to one-third

244 of that of a normal control at 21-years-of-age. Therefore, his clinical findings might show the early

245 phase of severe retinal degeneration. The reason for the differences of the progression between the

246 two siblings was not determined but some other genetic factors rather than environmental factors

247 might be responsible for this because these siblings had been raised in a similar environment. 
249 RPE [21], and they are assumed to be related to the remaining functional retina in eyes with

250 inherited retinal diseases [22, 23]. In the late stages of choroideremia, some patients have areas of

251 residual hyper-autofluorescence, called scalloped regions, surrounded by hypo-autofluorescent

252 areas [22, 23]. These hyper-autofluorescent areas are reported to be correlated with the areas of

253 surviving photoreceptors determined from multiple slices through the EZ in the OCT images [22,

254 23]. The size of these residual autofluorescent areas shrinks every year[24], and they have been

255 used to monitor the effectiveness of gene therapy in these types of patients [25]. Similar

256 autofluorescent areas were found in some eyes in our cohort. The high resolution AO fundus

257 images have allowed researchers to detect the subtle changes of the macular structures during the

258 course of retinal degeneration. Therefore, for the evaluation of the photoreceptor structure in

"scalloped regions" of choroideremia, our AO image analysis might be useful.

260 Our results showed that the course of reduction of the BCVA was composed of three phases. Most

patients had a reduction in the BCVA soon after the initial visit due to an involvement of the

263 of case NA209. However, the BCVA was relatively well preserved until the mid-20s, and then the

264 BCVA decreased in a relatively quickly (Fig. 2). The AO image analyses suggested that the

265 preserved photoreceptor islands were responsible for the maintained BCVA of $>0.1$ (decimal). The 
disruption of the photoreceptor islands appeared to lead to a further decrease in BCVA to nearly

complete blindness. Indeed, the FAF and AO images did not detect any photoreceptor islands in near blind eyes such as the NA0039Rt, NA1201Rt, and NA1209Rt and Lt. accurately determined mainly because of the limited number of studies of the high-resolution AO images. HRCs were found at the edge of photoreceptor islands but their origins are uncertain. derivatives. Some of the round HRCs observed in vivo in the degenerating retina with geographic atrophy were shown to be of similar in size and shape to ectopic RPE cells reported by histology [26]. If this is true, the detached RPE cells might migrate to the edge of the photoreceptor islands because the appearance and size of the HRCs in our study (Fig. 7) are identical to those in the previous report [26]. It is known that following the death of all photoreceptors, the RPE cells detach from Bruch's membrane and migrate to perivascular sites in the inner retina producing the bone spicule pigments [27]. It is possible that a similar migration of RPE is related to the loss of photoreceptor islands. 
addition, statistical analyses could not be performed due to the low number of images and different

285 times of obtaining the images. In addition, not only the size but also the quality of photoreceptor

286 island images would affect the BCVA because the BCVA of NA1201Lt was maintained at 0.5 in

287 spite of a decrease in the size of photoreceptor islands. Future investigations of the size and quality

288 of the photoreceptor islands using microperimetry might provide additional information that will

289 help in making a prognosis of RP patients.

290 Another limitation is"photoreceptor island" is not a common feature of the RP. Usually in the

291 advanced stage of RP, AO images can detect only mottled structures with unknown origin and

292 "photoreceptor island" were found in a small portion of the eyes with RP. Thus our AO image

293 analysis can be applied for the limited number of RP patients.

294 In conclusion, we have analyzed the phenotypic characteristics of arRP eyes carrying the $\mathrm{m} 1$

295 variant. These patients had severe retinal degeneration with early macular involvement as reported

296 in arRP eyes caused by other $\underline{R P 1}$ variants $[4,6,7,11,20]$. In the course of macular degeneration

297 in our cohort, the AO images showed not only the reduction of the cone intensity in one patient but

298 also showed the process of the reduction of the residual photoreceptor areas at the end stage of RP.

299 These changes indicated that total blindness appeared to be related to the loss of the photoreceptor

300 islands. Monitoring the fine retinal structures and the cone mosaics by AO fundus imaging could 
301 provide further prognostic implications and can be used for the evaluation of new therapeutic

302 applications.

303

304 


\section{Acknowledgements}

306 The authors thank Professor Emeritus Duco Hamasaki of the Bascom Palmer Eye Institute for the 307 discussions and editing the final version of the manuscript. This work was supported in part by the 308 Japan Society for the Promotion of Science (JSPS) KAKENHI Grant Numbers 19K09928 to S.U 309 and Takayanagi Retina Research Award to S.U. 


\section{References}

312 1. Pierce EA, Quinn T, Meehan T, McGee TL, Berson EL, Dryja TP. Mutations in a gene

313 encoding a new oxygen-regulated photoreceptor protein cause dominant retinitis pigmentosa. Nat

314 Genet 1999;22:248-54.

315 2. Sullivan LS, Heckenlively JR, Bowne SJ, Zuo J, Hide WA, Gal A et al. Mutations in a

316 novel retina-specific gene cause autosomal dominant retinitis pigmentosa. Nat Genet 1999;22:255-9.

317 3. Khaliq S, Abid A, Ismail M, Hameed A, Mohyuddin A, Lall P et al. Novel association of

318 RP1 gene mutations with autosomal recessive retinitis pigmentosa. $J$ Med Genet 2005;42:436-8.

319 4. Riazuddin SA, Zulfiqar F, Zhang QJ, Sergeev YV, Qazi ZA, Husnain T, Caruso R et al.

320 Autosomal recessive retinitis pigmentosa is associated with mutations in RP1 in three

321 consanguineous Pakistani families. Invest Ophthalmol Vis Sci 2005;46:2264-70.

322 5. Avila-Fernandez A, Corton M, Nishiguchi KM, Munoz-Sanz N, Benavides-Mori B,

323 Blanco-Kelly F et al. Identification of an RP1 prevalent founder mutation and related phenotype in

324 Spanish patients with early-onset autosomal recessive retinitis. Ophthalmology 2012;119:2616-21.

$3256 . \quad$ Kurata K, Hosono K, Hotta Y. Clinical and genetic findings of a Japanese patient with

326 RP1-related autosomal recessive retinitis pigmentosa. Doc Ophthalmol 2018;137:47-56. 
327 7. Verbakel SK, van Huet RAC, den Hollander AI, Geerlings MJ, Kersten E, Klevering BJ et

328 al. Macular dystrophy and cone-rod dystrophy caused by mutations in the RP1 gene: Extending the

329 RP1 disease spectrum. Invest Ophthalmol Vis Sci 2019;60:1192-203.

$330 \quad 8 . \quad$ Riera M, Abad-Morales V, Navarro R, Ruiz-Nogales S, Mendez-Vendrell P, Corcostegui B

331 et al. Expanding the retinal phenotype of RP1: from retinitis pigmentosa to a novel and singular

332 macular dystrophy. Br J Ophthalmol 2019. doi:10.1136/bjophthalmol-2018-313672

333 9. Liu Q, Zhou J, Daiger SP, Farber DB, Heckenlively JR, Smith JE et al. Identification and

334 subcellular localization of the RP1 protein in human and mouse photoreceptors. Invest Ophthalmol

335 Vis Sci 2002;43:22-32.

336 10. Liu Q, Lyubarsky A, Skalet JH, Pugh EN, Pierce EA et al. RP1 is required for the correct

337 stacking of outer segment discs. Invest Ophthalmol Vis Sci 2003;44:4171-83.

338 11. El Shamieh S, Boulanger-Scemama E, Lancelot M-E, Antonio A, Demontant V, Condroyer

$339 \mathrm{C}$ et al. Targeted next generation sequencing identifies novel mutations in RP1 as a relatively

340 common cause of autosomal recessive rod-cone dystrophy. BioMed Res Int 2015.

341 doi:10.1155/2015/485624

342 12. Nikopoulos K, Cisarova K, Quinodoz M, Koskiniemi-Kuendig H, Miyake N, Farinelli P et

343 al. A frequent variant in the Japanese population determines quasi-Mendelian inheritance of rare

344 retinal ciliopathy. Nat Commun 2019;10:2884. doi: 10.1038/s41467-019-10746-4. 
346 of cone photoreceptors in eyes with occult macular dystrophy. Invest Ophthalmol Vis Sci

$347 \quad 2015 ; 56: 7243-9$.

348 14. Ueno S, Kawano K, Ito Y, Ra E, Nakanishi A, Nagaya M et al. Near-infrared reflectance

349 imaging in eyes with acute zonal occult outer retinopathy. Retina 2015;35:1521-30.

350 15. Ueno S, Nakanishi A, Kominami T, Ito Y, Hayashi T, Yoshitake K et al. In vivo imaging of

351 a cone mosaic in a patient with achromatopsia associated with a GNAT2 variant. Jpn J Ophtalmol

$352 \quad 2017 ; 61: 92-8$.

353 16. Ueno S, Nakanishi A, Sayo A, Kominami T, Ito Y, Hayashi T et al. Differences in ocular

354 findings in two siblings: one with complete and other with incomplete achromatopsia. Doc

J Ophthalmol 2004;137:1137-39. 
363 Genetic characteristics of retinitis pigmentosa in 1204 Japanese patients. J Med Genet 2019; 56:

$364 \quad 662-70$.

365 20. Chen LJ, Lai TYY, Tam POS, Chiang SWY, Zhang X, Lam S et al. Compound

366 heterozygosity of two novel truncation mutations in RP1 causing autosomal recessive retinitis

367 pigmentosa. Invest Ophthalmol Vis Sci. 2010;51:2236-42.

368 21. Schmitz-Valckenberg S, Holz FG, Bird AC, Spaide RF. Fundus autofluorescence imaging:

369 review and perspectives. Retina 2008;28:385-409.

history of the central structural abnormalities in choroideremia: A prospective cross-sectional study. 
379 25. Xue K, Jolly JK, Barnard AR, Rudenko A, Salvetti AP, Patricio MI et al. Beneficial effects 380 on vision in patients undergoing retinal gene therapy for choroideremia. Nat Med 2018;24:1507-12.

381 26. Gocho K, Sarda V, Falah S, Sahel JA, Sennlaub F, Benchaboune M et al. Adaptive optics 382 imaging of geographic atrophy. Invest Ophthalmol Vis Sci 2013;54:3673-80.

383 27. Milam AH, Li ZY, Fariss RN. Histopathology of the human retina in retinitis pigmentosa. 384 Prog Retin Eye Res 1998;17:175-205.

385 


\begin{tabular}{|c|c|c|c|c|c|c|c|c|c|c|c|}
\hline & 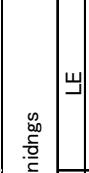 & 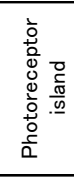 & $\mathbb{z}$ & 旁章 & 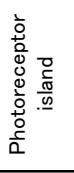 & 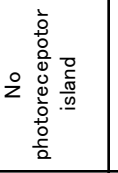 & 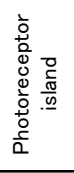 & 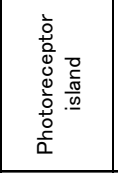 & 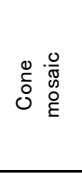 & 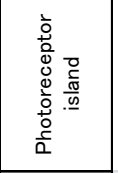 & \\
\hline & 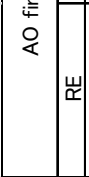 & 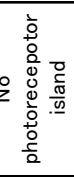 & $\underline{z}$ & 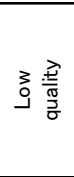 & 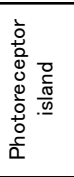 & 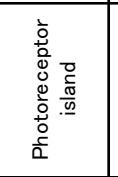 & 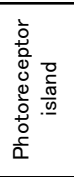 & 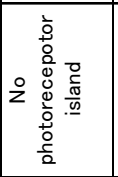 & 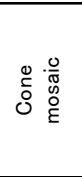 & 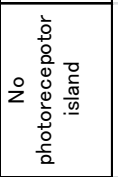 & \\
\hline \multirow{14}{*}{ 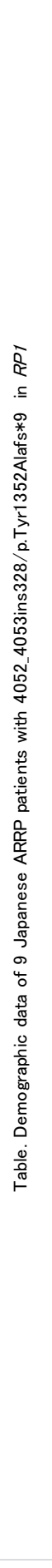 } & 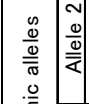 & $\bar{\varepsilon}$ & $\bar{E}$ & $\bar{\varepsilon}$ & $\bar{\varepsilon}$ & $\bar{\varepsilon}$ & $\tilde{\Xi}$ & $\tilde{\varepsilon}$ & $\tilde{\xi}$ & $\tilde{\varepsilon}$ & \multirow{14}{*}{ 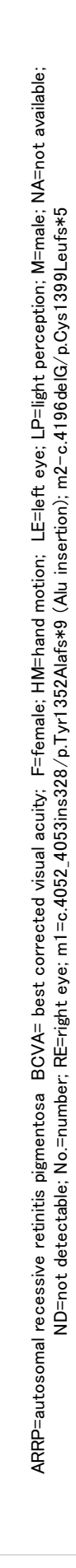 } \\
\hline & & $\bar{\varepsilon}$ & $\bar{\varepsilon}$ & $\bar{\varepsilon}$ & $\bar{\varepsilon}$ & $\bar{\varepsilon}$ & $\bar{\varepsilon}$ & $\bar{\varepsilon}$ & $\bar{\varepsilon}$ & $\bar{\varepsilon}$ & \\
\hline & 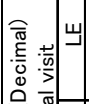 & ö & $\stackrel{+}{0}$ & ָ̃ & : & ̊̊ & $\overline{0}$ & $\stackrel{\circ}{\circ}$ & $\stackrel{\circ}{\circ}$ & Õ & \\
\hline & 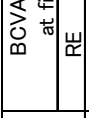 & $\stackrel{9}{ }$ & $\stackrel{\circ}{\circ}$ & 蔗 & 용 & $\stackrel{\text { L̊: }}{0}$ & æ⿱亠䒑 & æ⿱亠乂 & $\stackrel{\infty}{\circ}$ & æ⿱亠䒑 & \\
\hline & 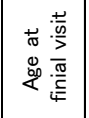 & ర్ల & ల్ల & $\stackrel{\theta}{-}$ & N & ஜू & $\tilde{ల}$ & $\ddot{\infty}$ & $\stackrel{N}{\circ}$ & \% & \\
\hline & 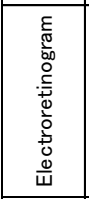 & $\hat{z}$ & $\stackrel{\mathbb{z}}{z}$ & z & $\hat{z}$ & 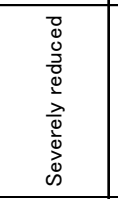 & $\bar{z}$ & z & 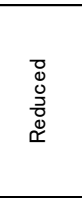 & z & \\
\hline & 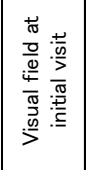 & 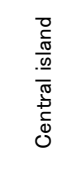 & 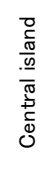 & 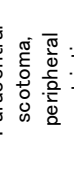 & 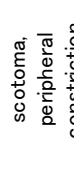 & 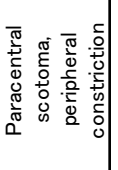 & 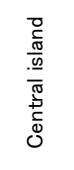 & 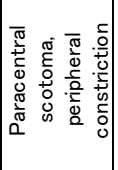 & 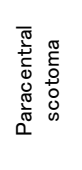 & 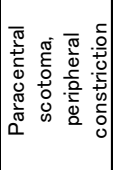 & \\
\hline & |l: & $\stackrel{\infty}{\circ}$ & $\stackrel{\circ}{\circ}$ & ó & $\stackrel{\circ}{\circ}$ & 号 & : & $\stackrel{\infty}{\infty}$ & - & ó & \\
\hline & 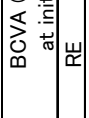 & $\stackrel{\infty}{\circ}$ & $\stackrel{\circ}{\circ}$ & $\stackrel{\circ}{\circ}$ & $\stackrel{\circ}{\circ}$ & $\stackrel{+}{\circ}$ & $\stackrel{\circ}{\circ}$ & $\stackrel{\infty}{\infty}$ & - & ث & \\
\hline & 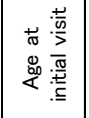 & $\bar{N}$ & $\bar{N}$ & $\simeq$ & \pm & $\stackrel{\llcorner}{\sim}$ & $\stackrel{2}{2}$ & $\infty$ & $\stackrel{2}{ }$ & $\stackrel{9}{-}$ & \\
\hline & 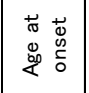 & 0 & 0 & ص & ๑ & 0 & 0 & 0 & $\stackrel{2}{2}$ & 0 & \\
\hline & ڤ્ & ч & $\Sigma$ & ᄂ & $\Sigma$ & ᄂ & $\Sigma$ & ч & $\Sigma$ & 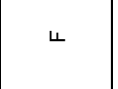 & \\
\hline & 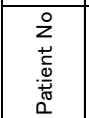 & $\begin{array}{l}\text { 兽 } \\
\frac{8}{2}\end{array}$ & $\frac{\mathscr{O}}{\frac{0}{\sigma}}$ & $\begin{array}{l}\text { ơ } \\
\text { ờ } \\
\frac{8}{2}\end{array}$ & $\begin{array}{l}\frac{\infty}{0} \\
\frac{1}{\frac{\pi}{z}} \\
\frac{1}{z}\end{array}$ & $\begin{array}{l}\stackrel{2}{0} \\
0 \\
\frac{1}{2}\end{array}$ & 啇 & 商 & 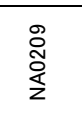 & 竞 & \\
\hline & 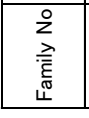 & - & - & $\sim$ & $\sim$ & m & $\sigma$ & $\sigma$ & ๘ & ๘ & \\
\hline
\end{tabular}


Figure 1. Pedigree charts of 9 autosomal recessive retinitis pigmentosa cases carrying the $\mathrm{m} 1$

variant (c.4052 4053ins328/p.Tyr1352Alafs*9) in the $R P 1$ gene.

390 The $\mathrm{m} 2$ variant (c.4196delG/p.Cys1399Leufs*5) was found in 2 families. There was no reported

391 consanguinity in any of these pedigrees. Probands are shown by the arrows.

Figure 2. Graph showing the course of the best-corrected visual acuity over time in all eyes

from our cohort.

$\underline{\text { Snellen visual acuity was converted to the logarithm of the minimum angle of resolution }}$

(logMAR). R; right L; left

Figure 3. Fundus color photographs of the right eye of the 9 patients from 5 families (1-5)

with autosomal recessive retinitis pigmentosa caused by a variant of the $R P 1$ gene;

400

c.4052_4053ins328/p.Tyr1352Alafs*9 (m1).

401 The age of the patients at which the photograph was taken is shown. All the patients except NA0209 

(1-5). patchy and coalescent hypo-autofluorescent areas including macula. Y.O. ; year old . Figure 5. Multimodal images in case NA0209.

a. Changes of retinal images during the follow-up period. Fundus autofluorescence (FAF, upper)

411 shows fine hypo-autofluorescent patchy spots around the arcade vessels and at the fovea appeared

412 during 5 years. The OCT images show a marked reduction of the retinal thickness in 5 years. Arrows

413 indicate the residual EZ at 20-years-of-age which disappeared in 5 years. The scale bar is $200 \mu \mathrm{m}$.

414 b. Adaptive optics (AO) fundus images of case NA0209 at 21-years-of-age. A montage image is

415 shown on the left. Magnified images of the white square (at 3 degrees from the central fovea on the temporal side and 1 and 3 degrees on the nasal side; shown as T3, N1 and N3 respectively) in the montage image are shown. For comparison the $\mathrm{AO}$ images of a normal control at 3 degrees from magnified AO images as well. 
421 Figure 6. Optical coherence tomographic (OCT) images of the right eye of the 9 patients from 5

422 families (1-5). The OCT images shows thinning of the retina and abnormalities in the outer retina in

423 all cases. Several fine hyperreflective structures can be seen on the RPE shown by the yellow

424 arrows.

425

Figure 7. Progressive reduction of surviving photoreceptor areas (photoreceptor islands) in the

right eye of case NA0201.

428

Fundus photograph (top left) shows a deep colored area in the central macula which was detected as

429

a hyper autofluorescence area in fundus autofluorescence (FAF, top middle) images. The white

430

squares in the color fundus photograph and FAF area corresponds to fundus AO images below.

431 Successive AO images were recorded on June 2013, January 2015, and January 2017. The OCT

images obtained on the same days are shown on the right of each AO fundus image, and the scanned

area is shown by the dotted arrows in the AO fundus images. Illustrative images of the photoreceptor

islands are shown at the bottom left of each AO image. The size of photoreceptor islands decreases

435

progressively. The magnified image of the white square in the AO fundus image on June 2013 is

436 shown at the top right. The edge of the photoreceptor island is partially covered by hyporeflective

437

clumps (HRCs). Small HRCs participate to form the large clumps. HRCs surrounding the 
in arrow heads. autofluorescence (FAF; top right), AO fundus image, and OCT image of each eye are shown. The area surrounded by the white line in the color fundus and FAF images correspond to the montage of the AO fundus images (middle). The first recorded AO fundus images of each patient (baseline of analysis) and OCT images (below) on the same day are shown. The scanned areas for the OCT images are shown by the yellow dotted arrows in the AO fundus images. The fundus and FAF images obtained on the closest day to the AO images are shown. Illustrations of the photoreceptor islands are shown at the upper left of each AO fundus image. Hyper autofluorescence areas in the central macula detected in FAF are clearly visible in the AO fundus images. each time point is also shown. 


\section{Family 1}

Family 2
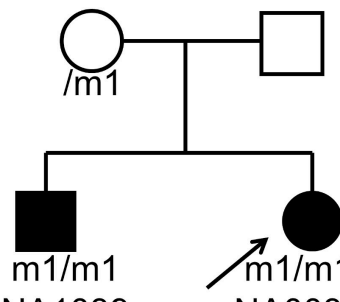

NA1039

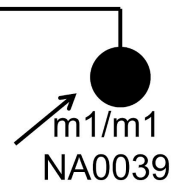

Family 4

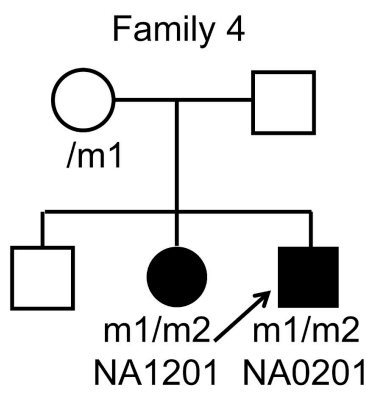

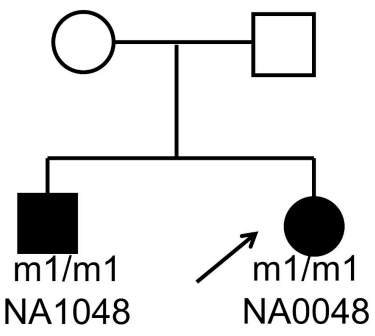

Family 5

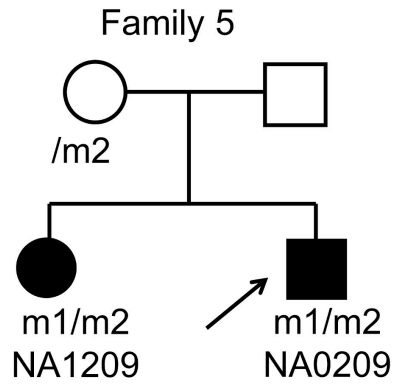

Family 3

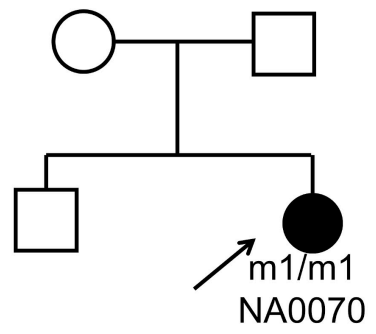


Visual acuity (LogMAR)

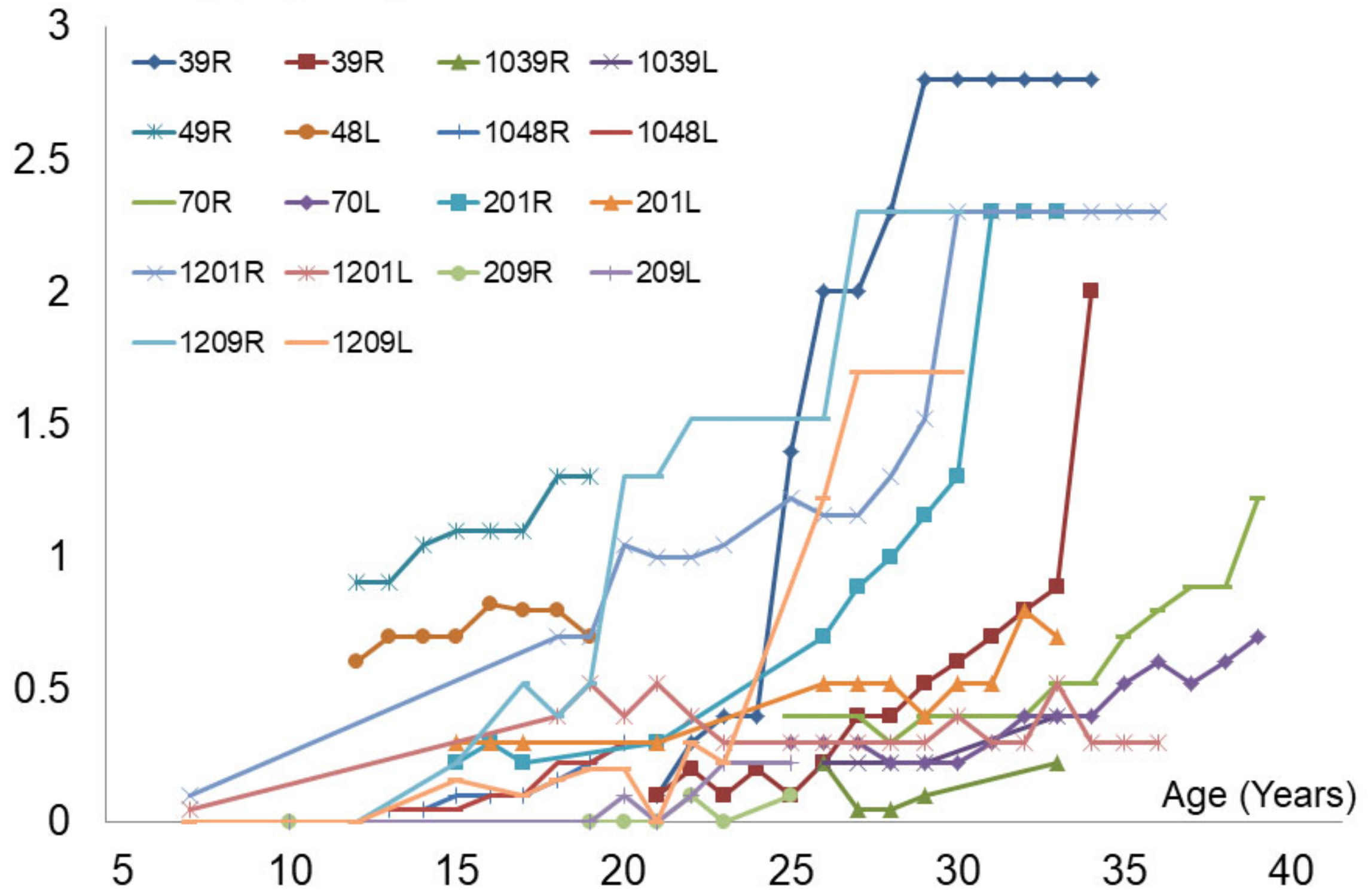

Graph showing the course of the best-corrected visual acuity over time in all eyes from our cohort. Snellen visual acuity was converted to the logarithm of the minimum angle of resolution (LogMAR). R; right L; left 
NA0039 29Y.O.

NA0048 19Y.O.
NA1039 33Y.O.

NA1048 18Y.O.

NA0070 25Y.O.

NA0201 26Y.O.

NA1201 29Y.O.

5

NA0209 20Y.O.

NA1209 26Y.O. 


\section{NA0039 31Y.O.}

NA0048 16Y.O.

NA1048 18Y.O.

NA0070 36Y.O.

NA0201 29Y.O.

NA1201 33Y.O.

NA0209 21Y.O.

NA1209 26Y.O. 

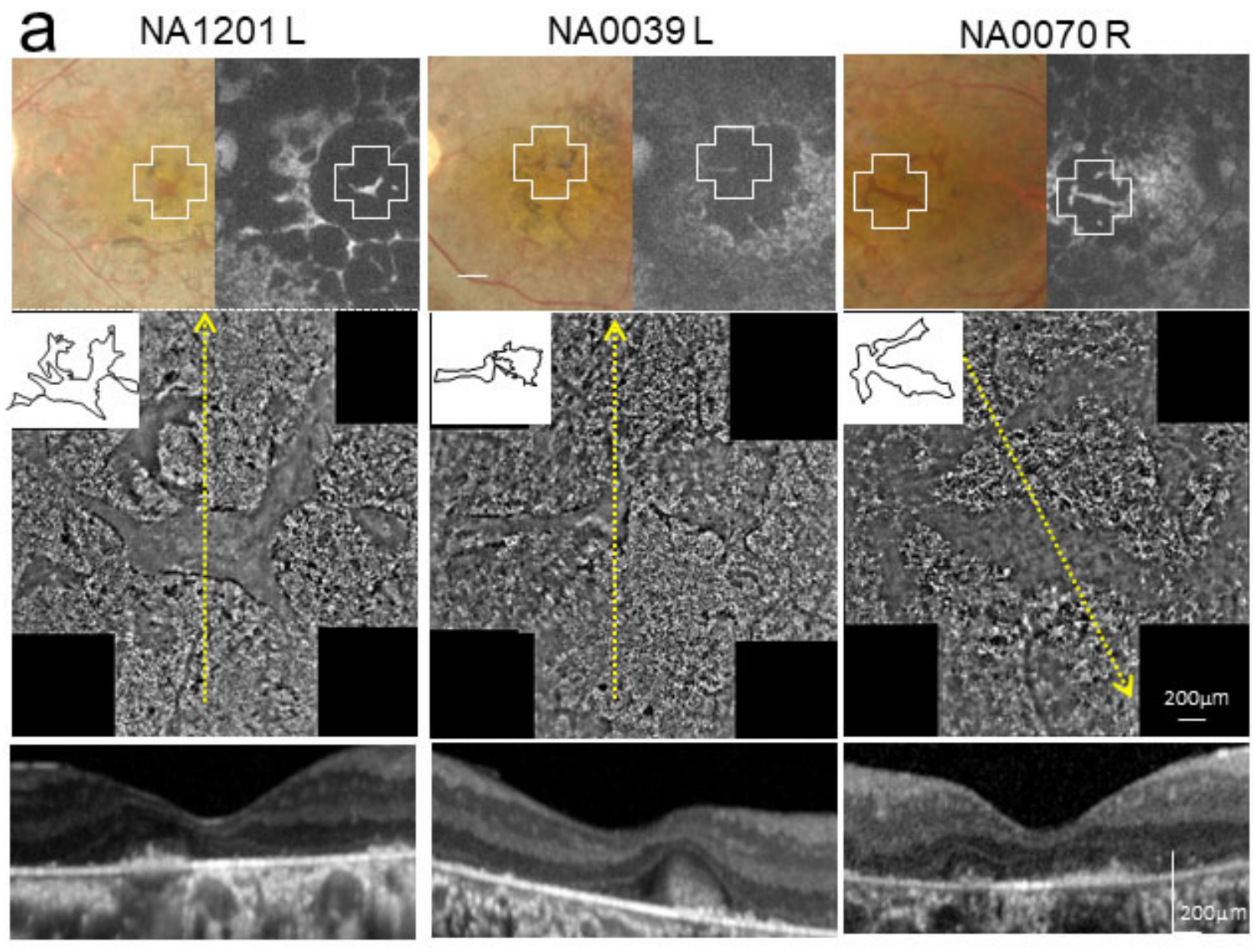

$b^{\text {Photoreceptor area }}$

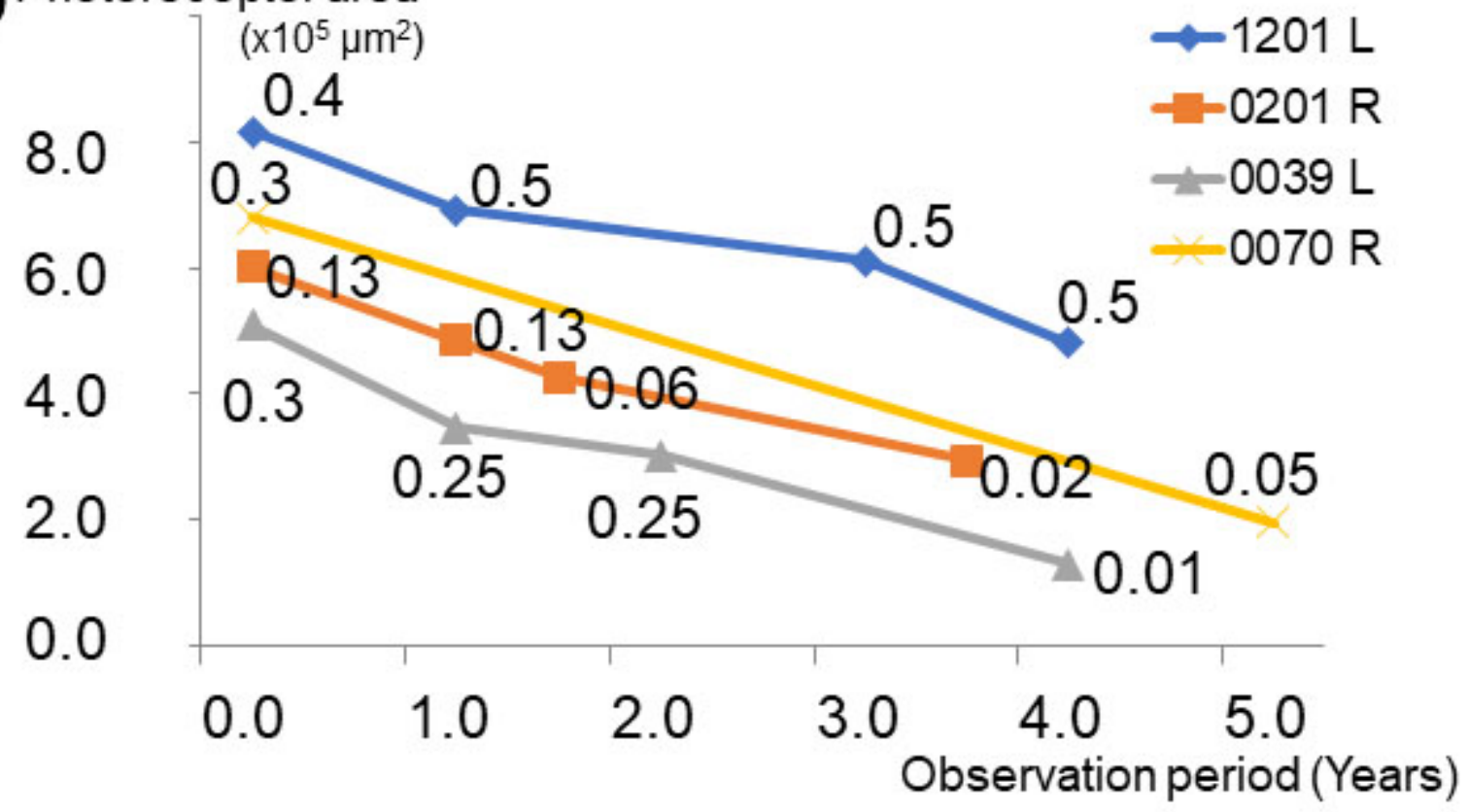




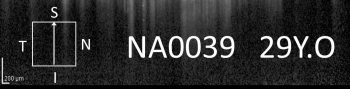

2
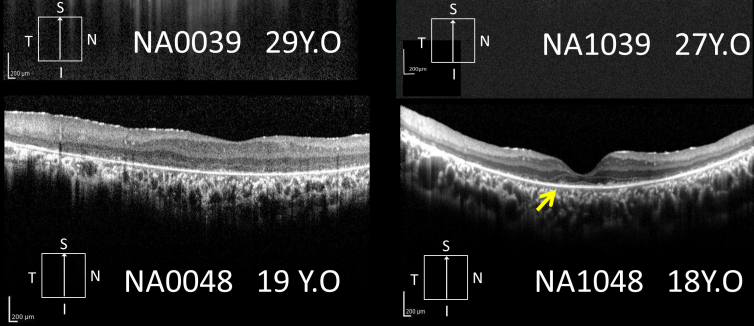

3
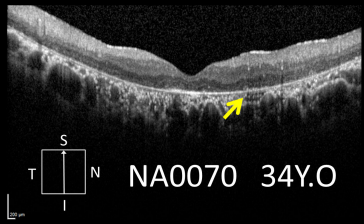

4
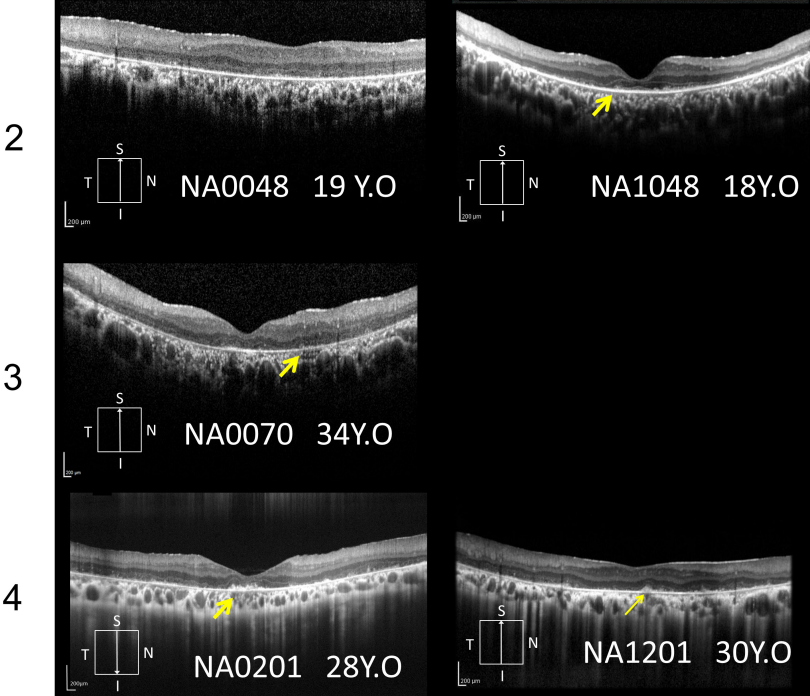

5
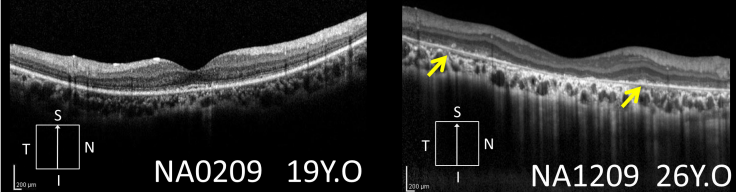


\section{a $\quad N A 1201 \mathrm{~L}$}

NA0039 L
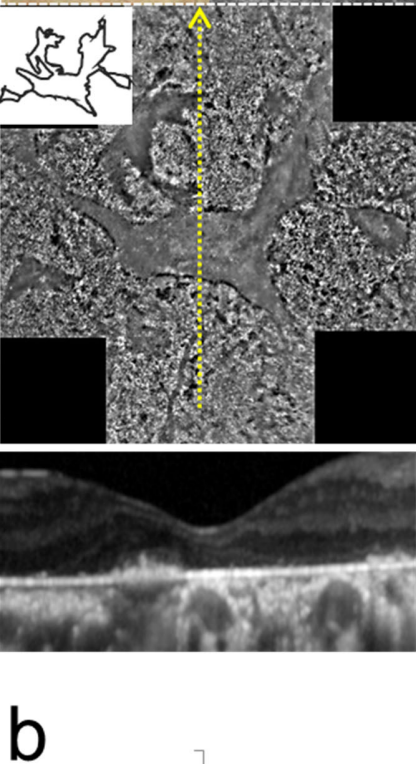

b

$\left(\mathrm{x} 10^{5} \mu \mathrm{m}^{2}\right)$

8.0

6.0

4.0

2.0

0.0
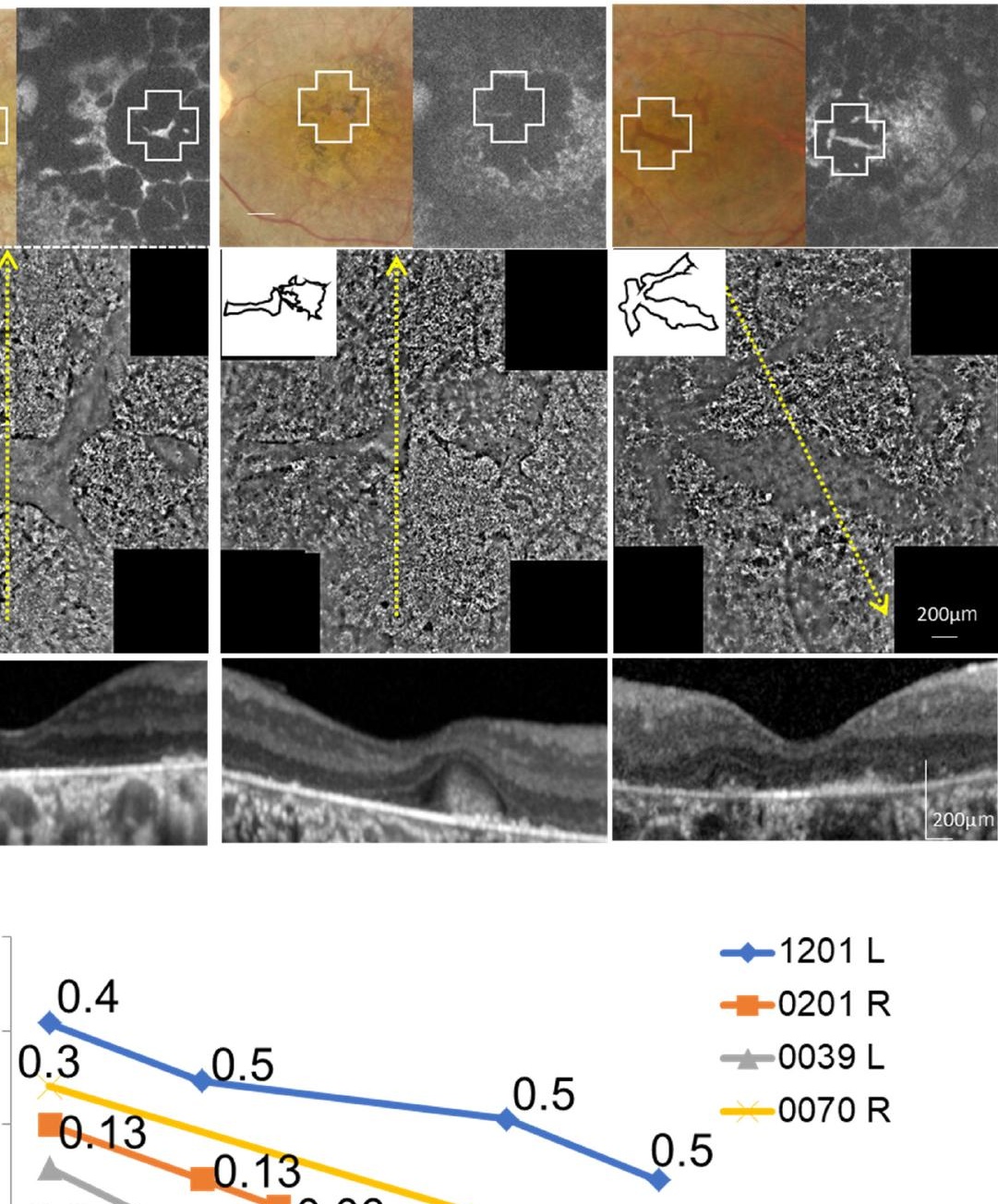

NA0070 R 